\title{
Random control procedures in classical skin conductance conditioning
}

\author{
WILLIAM F. PROKASY \\ University of Utah, Salt Lake City, Utah 84112
}

\begin{abstract}
There have been differences in opinion concerning what constitute proper procedures for providing random controls in classical skin conductance conditioning. The present paper is a comment on selected aspects of a methodological discussion of control procedures and permissable inferences provided by Furedy, Poulos, and Schiffman (1975).
\end{abstract}

The classical conditioning situation is one which involves an array of event-event and event-time conditional relationships, or contingencies (Prokasy, 1965). Given an environment in which the likelihood of an aversive event (UCS) per unit time is greater than zero, the likelihood may vary conditional upon the presence or absence of another stimulus or signal. Thus, a CS+ is a stimulus for which the conditional likelihood of the UCS is greater than it is for a control baseline. Similarly, A CS- is a stimulus for which the conditional likelihood of the UCS is typically zero.

The most common arrangement in single-cue, or simple, conditioning is one in which one group of subjects receives a pairing of CS and UCS and in which the other receives a mixed, unpaired ordering of the same number of CSs and UCSs. The likelihood of the UCS in the $a b$ sence of the CS is zero. Within-groups contrast is obtained when a single group receives CS+, UCS pairing, and a CS - in an unpredictable order. As in single-cue training, the likelihood of the UCS in the absence of either signal is zero.

In both single-and double-cue arrangements, the control baseline is assumed to be the response level to the signal which is explicitly unpaired with the UCS, and the amount of conditioning is defined as the difference between that to the control stimulus and that to the CS+. Depending upon one's theoretical vantage point, the difference may be attributable to excitation, to inhibition, or both.

There is an operational distinction between the singleand double-cue conditioning arrangments which, from a theoretical point of view, may allow different combinations of excitation and inhibition to be manifest. As noted earlier (Prokasy, 1965), the typical control group is composed of subjects who receive an unpredictable UCS in the absence of a signal and who receive a CSwhich has no UCS paired with it. Thus, $\mathrm{P}\left(\mathrm{UCS} / \mathrm{CS}_{-}\right)$is less than $\mathrm{P}(\mathrm{UCS} / \overline{\mathrm{CS}}-)$. This inequality contrasts with that of the two-cue situation in which $\mathrm{P}(\mathrm{UCS} / \mathrm{CS})=$ $\mathrm{P}(\overline{\mathrm{USS}} / \overline{\mathrm{CS}}+)$. The distinction is in the background stimulation. For the single-cue sequential control, the

Supported by NIMH Grant MH-15 353. background stimulation includes unpredictable UCSs. It is an environmental setting in which aversive stimuli are present in uncued fashion. For differential conditioning, the background does not include unpredictable UCSs.

Rescorla (1967) theorized and demonstrated with a conditioned suppression paradigm that, when P(UCS/ $\mathrm{CS}-$ ) is less than $\mathrm{P}(\mathrm{UCS} / \mathrm{CS}-)$, the $\mathrm{CS}-$ becomes inhibitory. Thus, it became of theoretical and methodological interest to determine more generally whether or not the typical single-cue conditioning situation is one which involves a contrast including both excitatory and inhibitory influences. Toward that end, several studies (Fureday, 1971, 1974; Furedy \& Schiffman, 1971, 1973; Prokasy, Williams, Kumpfer, Lee, \& Jenson, 1973; and Schiffman \& Furedy, 1972) have been designed to determine whether or not skin conductance conditioning to a signal differs depending upon whether or not the likelihood of the UCS in the absence of that signal is less than or equal to the likelihood of the UCS in the $a b$ sence of any signal. For present purposes, "CS -" will refer to a signal for which there is no overlap of signal and UCS, and "RS" (random signal) will refer to a signal for which the likelihood of the UCS is the same both in the presence and absence of that signal. It is to be emphasi-zed that none of the cited studies as designed could determine whether or not the CS- acquired inhibitory properties. At best, they constitute a first step of determining whether or not skin conductance response (SCR) conditioning is differentially sensitive to two signals, a CS - and an RS, under circumstances in which the likelihood of an unpredictable UCS as part of the background stimulation is greater than zero.

Of the cited studies, only that of Prokasy et al. (1973) provided some evidence that SCR performance to CS - is less than that to RS. The evidence is quite limited, however, and requires a distinction between tirst-interval responses (FIRs) and second-interval responses (SIRs). The FIR latency range was defined as 1.2 to $3.7 \mathrm{sec}$ after CS onset, and the SIR latency range was defined as 3.7 to $6.2 \mathrm{sec}$ after CS onset. Neither frequency nor amplitude of FIRs differed to CS+ and RS, although frequency was less to CS- than to either 
the other two stimuli. SIR amplitude decreased reliably from $\mathrm{CS}+$ to $\mathrm{CS}-$. The frequency effect paralleled the amplitude outcome, but statistically reliable effects were not uniformly obtained, the outcome depending upon whether or not the data employed were from all subjects or from those who met a minimum response frequency. Two conclusions were made. First, it was concluded that, since the FIR and SIR outcomes did not parallel each other, it is difficult to interpre! the results in terms of general excitatory or inhibitory influences. Second, it was concluded that, since the FIR, as an orienting response, is elicited with signal onset, the SIR might provide a better index for relative excitation and inhibition. If so, one might in turn argue "that the CS+ acquires excitatory properties and the CS.. acquires inhibitory properties as measured with the SIR." The relative neutrality of the RS can be defended on the grounds that response frequency and amplitude to that stimulus equalled frequency and amplitude of nonspecific responses (i.e., responses occurting in the absence of experimenter-presented stimuli).

In contrasting their findings with those of the then available Toronto studies (Furedy, 1971; Furedy \& Schiffman, 1973), Prokasy et al. (1973) drew several contrasts: the Toronto studies employed a between. subjects comparison, the Utah study a within-subjects comparison; the Toronto studies report only FIRS, the Utah study both FIRs and SIRs; and the Toronto studies incorporate a RS which is more like a CS-than a signal presented randomly with respect to background UCS occurrence.

In a recent paper, Furedy, Poulos, and Schiffman (1975) discussed some general problems of methodology and inference in SCR conditioning with specific reference to the randomization procedures and conclusions in the Toronto and the Utah studies. It is the purpose of this paper to respond to several aspects of the Furedy et al. article.

\section{THE UTAH STUDY}

Concerning the Utah study, Furedy et al. (1975) state: 'Within the contingency formulations the 'RS' ... meets the specification of an excitatory CS since UCS occurrence is more probable in the presence of the RS than in its absence..." (italics theirs). In order to de termine whether or not this is the case, a summary of the operations of the Utah study is necessary. The experimental period of 46 min was divided into 540 $5.1-\mathrm{sec}$ time units. A $5-\mathrm{sec}$ event (one of three stimuli) was assigned at a likelihood of .25 per time unit randomly throughout the session, this resulting in a total of 134 such events. The events were randomly assigned as either CS+ (44 instances), CS- (44 instances), and RS (43 instances). Then the experimental period was divided into $27001.2 \mathrm{sec}$ units. A UCS was assigned with likelihood .033 to these units, with the result that 95

\begin{tabular}{lccc}
\multicolumn{4}{c}{ Table 1} \\
\\
\cline { 2 - 3 } Event Catcgory & Total Sec & Total UCS & UCS/Sec \\
\hline CS+ & 220 & 21 & .095 \\
CS - & 220 & 0 & .000 \\
RS & 215 & 4 & .019 \\
No signal on & 2099 & 56 & .027 \\
No signal less dead space* & 1395 & 56 & .040 \\
Total time less RS & 2539 & 77 & .030 \\
Intire session & 2754 & 81 & .029
\end{tabular}

*ight-second periods following CSt and CS-; see text.

UCSs were generated for the 46-min period. Adjustments were made (see details in Prokasy et al., 1973) which resulted in a CS+ having no UCS occur within 13 sec of its onset, and the random relationship between KS and UCS remaining as randomly generated. Since UCSs were dropped to create a CS--, the number of remaining UCSs was 81 .

Table 1 summarizes the situation. The first column specifies the event category, with the total number of seconds devoted to that category provided in Column 2. The total number of UCSs occurring during each event category and its resulting rate-per-second are in the remaining columns. It should be observed that the 21 UCSs in the CSt category occurred at the end of the $5-\sec$ CS + . In addition, the category "no signal less dead space" refers to the fact that for $8 \mathrm{sec}$ following the offsets of CSt and CS - no UCSs occurred. An exception to this last rule was that if a second CS had been designated to occur within $8 \mathrm{sec}$ of the offset of an earlier one, the UCS rule pertaining to the second was in force. The RS was reinforced $9.3 \%$ of the time, but the instances of reinforcement occurred at any point during the time of the RS; i.e., there was no temporal contingency between RS and UCS.

The column in Table 1 of particular interest is the last one. No matter on what basis one calculates the back. ground rate of UCS occurrence, the rate of occurrence in the presence of RS is less. The only UCS rate which is less than that to the RS is that to the CS-, which is precisely what was intended by the design. Furedy et al. (1975) are wrong when they state that "... US occurrence is more probable in the presence of the RS than in its absence ..." These rates of UCS occurrence also imply that performance differences between RS and CS-cannot be attributed to an artifact of RS being excitatory with respect to background stimulation.

\section{THE TORONTO STUDIES}

Furedy et al. (1975) concluded that the RS in the Toronto studies did meet the specification of being random in its relationship to the UCS. They also alleged that the contrasting conclusion made by Prokasy et al. (1973) to the effect that the RS in those studies was more like a CS-- "derives directly from an inappropriate 
Table 2

Number of UCSs in Three Successive Time Blocks Beginning With RS Onset

\begin{tabular}{llcr}
\hline \multicolumn{1}{c}{ Study } & \multicolumn{3}{c}{ Time Block } \\
& 1 & 2 & 3 \\
\hline Furedy (1971), Experiment 1 & 0 & 4 & 7 \\
Furedy (1971), Experiment 2 & 1 & 5 & 6 \\
Furedy and Schiffmann (1971) & 3 & 3 & 0 \\
Schiffmann and Furedy (1972) & 2 & 3 & 5 \\
Furedy and Schiffmann (1973) & 2 & 4 & 5 \\
Furedy (1974), Experiments 1,2,3 & 2 & 4 & 0 \\
Furedy (1974), Experiment 4 & 2 & 4 & 0 \\
\hline
\end{tabular}

application of a traditional pairings analysis to the notion of randomness" (italics theirs).

The Toronto studies were all conducted in a common format involving two groups of subjects. One group received CS+ and CS-, the other CSt and RS. It is the latter group in each study which is of present concern. The UCSs were distributed in non-CSt periods and usually totaled 15 in number. The time periods following RS onset were divided into three equal parts of either 10- or 20-sec durations, and the number of the UCSs assigned to each part is provided in Table $2 .^{1}$ There were additional UCSs occurring between trials at longer time spans than the 30 (or 60) sec following RS onset, but they are not important for present purposes. The first three rows of Table 2 include data from the two papers available at the time the Utah study was submitted for publication.

If the background UCSs in the Toronto studies were distributed randomly over the three intervals, then onethird of them would be expected to occur in the first interval, the one which contains the RS. Of the total of 29 UCSs in the first three lines of Table 2, only four, instead of the expected 9.67, occurred in the first of the three time intervals. This is a reliable downward bias, $\chi(1)=5.07$. The UCSs were distributed less densely in the 10 (or 20) sec interval which contained the RS than in intervals which followed subsequently, thus making the RS more like a CS-; this bias does not exist uniformly in all the Toronto studies, but it does take an extreme form in the first study listed in Table 2: not a single UCS for any subject occurred within $10 \mathrm{sec}$ of RS onset.

The biases in the Toronto studies go beyond that of the distribution of between-trials UCSs, however. Those UCSs which fell within the 10 (or 20) sec period just following RS onset were assigned at random to one of the 20 (or 40) $.5-\mathrm{sec}$ time units composing the period. The location of the UCSs was randomized separately for each subject, which means that a subject may or may not have experienced an overlap of RS and UCS. Given random assignment of the UCS within the time interval containing the RS, one can calculate the expected proportion of time that the RS and UCS failed to occupy a common time unit throughout an entire session. These expectations are provided in Table 3. Since a

different random sequence was generated for each subject, it follows that the proportions in Table 3 are also the expected proportions of subjects who did not experience a chance RS-UCS overlap. ${ }^{2}$ Subjects not receiving a chance RS-UCS overlap have, for all practical purposes, received a CS-, not an RS, since for them the empirical likelihood of RS-UCS overlap is zero as it is for a CS-. With the exception of one recent study, the proportions are sizable. Thus, not only are some studies biased with a low UCS frequency in the immediately following vicinity of the RS, sizable proportions of subjects received, effectively, CS- instead of RS.

Another difficulty with the Toronto studies is their heavy reliance on a very low between-trials UCS rate. With usually only 15 UCSs distributed across an experimental session of $20 \mathrm{~min}$ or more, for 6 of the 9 experiments a single chance overlap of RS and UCS provided comparable UCS contingencies for the RS and background. For one of the studies (Furedy \& Schiffman, 1971), even one chance overlap of RS and UCS would make the empirical conditional likelihood of a UCS in the presence of the RS roughly eight times as great as its likelihood per unit time in the absence of any stimuli. For the remaining two studies UCS likelihoods in the presence and absence of the RS were equivalent with two chance overlaps of RS and UCS. Departures from the values of one and two chance overlaps produce shifts in the UCS rate per unit time which are on the order of two to one or greater with respect to background. Table 4 summarizes for the Toronto studies what the empirical rate-per-unit time is for the UCSs in the presence and absence of the RS given different numbers of RS-UCS chance overlap. The magnitude of change in the rate of UCS per unit time to the RS with a shift of but one chance overlap is sizable. From an individual subject point of view, it is difficult to understand what it means to base a contingency on an expected one or two overlaps of RS with UCS. For those subjects who did receive an overlap, it could have come at any point in training. Obviously the later in training it occurred, the more the data employed in making comparisons would reflect the influence of CS- rather than RS.

Whether or not a CS- acquires inhibitory properties remains an open question, and it may well be that further research will be consistent with the outcome of

Table 3

Expected Proportion of Reinforcement Schedules With No Chance Overlap of RS and UCS in the Toronto Studies

Study

Furedy (1971), Experiment 1

Furedy (1971), Experiment 2

Furedy and Schiffmann (1971)

Schiffmann and Furedy (1972)

Furedy and Schiffmann (1973)

Furedy (1974), Experiments 1, 2, 3

Furedy (1974), Experiment 4
Proportion

1.00

.20

.73

.36

.46

.25

.02 
Table 4

Empirical UCS Rates Per Second for Both Background and RS Durations as a Function of Different Numbers of Chance RS-UCS Overlaps

\begin{tabular}{|c|c|c|c|c|c|}
\hline Study & Stimulus & 0 & $\begin{array}{c}\text { Number of Chance } \\
1\end{array}$ & $\begin{array}{l}\text { RS-UCS Overlaps } \\
2\end{array}$ & 3 \\
\hline $\begin{array}{l}\text { Furedy (1971), Experiment } 1 \\
\text { Furedy and Schiffmann (1973) } \\
\text { Furedy (1974), Experiment } 4\end{array}$ & $\begin{array}{l}\text { Background } \\
\text { RS }\end{array}$ & $\begin{array}{l}.014 \\
.000\end{array}$ & $\begin{array}{l}.013^{*} \\
.013^{*}\end{array}$ & $\begin{array}{l}.012 \\
.027\end{array}$ & $\begin{array}{l}.011 \\
.040\end{array}$ \\
\hline Furedy (1971), Experiment 2 & $\begin{array}{l}\text { Rackground } \\
\text { RS }\end{array}$ & $\begin{array}{l}.016 \\
.000\end{array}$ & $\begin{array}{l}.015 \\
.007\end{array}$ & $\begin{array}{l}.014^{*} \\
.014^{*}\end{array}$ & $\begin{array}{l}.013 \\
.020\end{array}$ \\
\hline Furedy and Schiffmann (1971) & $\begin{array}{l}\text { Background } \\
\text { RS }\end{array}$ & $\begin{array}{l}.013 \\
.000\end{array}$ & $\begin{array}{l}.012 \\
.093\end{array}$ & $\begin{array}{l}.011 \\
.186\end{array}$ & $\begin{array}{l}.010 \\
.279\end{array}$ \\
\hline Schiffmann and Furedy (1972) & $\begin{array}{l}\text { Background } \\
\text { RS }\end{array}$ & $\begin{array}{l}.016 \\
.000\end{array}$ & $\begin{array}{l}.015 \\
.008\end{array}$ & $\begin{array}{l}.014^{*} \\
.017^{*}\end{array}$ & $\begin{array}{l}.013 \\
.025\end{array}$ \\
\hline Furedy (1974), Experiments 1, 2, 3 & $\begin{array}{l}\text { Background } \\
\text { RS }\end{array}$ & $\begin{array}{l}.014 \\
.000\end{array}$ & $\begin{array}{l}.013^{*} \\
.017^{*}\end{array}$ & $\begin{array}{l}.012 \\
.033\end{array}$ & $\begin{array}{l}.011 \\
.050\end{array}$ \\
\hline
\end{tabular}

*These overlap combinations are the only ones that provide an approximate equality between UCS likelihood in the presence and absence of the RS.

the Toronto studies. However, the Toronto studies do not constitute an adequate basis on which to conclude that there are no inhibitory effects because randomization requirements were not met. In addition, the present view that the RS in those studies is more like a CS- has nothing to do with an alleged inappropriate application of a pairings analysis; rather it follows from what appear to be design inadequacies in meeting contingency requirements.

\section{SUBJECTIVE CONTINGENCIES}

Furedy and his associates have been concerned with whether or not the "truly random" control is "appropriate" for SCR conditioning. As part of their effort, they have made several attempts to assess the subjective likelihoods of UCS occurrence which subjects assign to each stimulus. It is not the present purpose to evaluate those attempts, but as regards identifying subjective contingeny, Furedy et al. (1975) go on to say: "That sort of identification was attempted by Prokasy et al. (1973) in their human-based study, but neither their procedures nor their method of gathering those aspects of the data seem optimal." That comment creates the highly misleading impression that one intention of the Utah study was to assess subjective contingencies. That was patently not the case. The subjects in the Utah study were informed of the contingencies as part of the instructions and were asked, following the session, to identify which contingency was associated with which signal. The objective was simply to assure that subjects could identify which stimulus was which among the CS,$+ \mathrm{RS}$, and $\mathrm{CS}-$.

\section{THE PAIRINGS ANALYSIS}

Furedy et al. (1975) correctly observe that obtaining a higher performance level to RS than to CS-does not logically require the conclusion that the $\mathrm{CS}-$ has acquired inhibitory properties. It might be argued, for exam- ple, that RS performance is simply more excitatory than CS-performance, this reflecting a difference between the effects of a reinforcement schedule with a low reinforcement ratio and a schedule of no reinforcement at all. Short of additional research, whether or not the Utah study constitutes a clear example of inhibition remains to be seen. It is to be noted, however, that not only was CS - performance less than RS performance with the SIR as the measure, it was less than performance obtained with behavior sampled during the absence of experimenter-presented stimulation. Thus, CSperformance was less than background performance, an effect which follows clearly from the view that inhibitory effects are present but which does not follow quite as simply from the idea that only excitatory effects are present and are induced strictly via CS.UCS pairings.

In contrasting what they call the "contingency" theory and the "pairings" theory, Furedy et al. (1975) note that RS performance being greater than CS- performance does not eliminate a "pairings" interpretation and that further tests are necessary. They err, however, when they state that when no differences are obtained "no further steps need be taken along such lines." There are at least two issues at stake: do the experimental operations result in active inhibition and is the measure taken one which can reflect, directly, inhibitory influences? The failure to obtain RS, CS- differences may tell us no more than that the measure itself is insensitive to active inhibitory effects and that only subsequent tests (such as summation tests) can differentiate the options. Thus, subsequent tests are necessary regardless of whether or not initial differences exist.

\section{RESPONSE DEFINITION}

In one major section of their paper, Furedy et al. (1975) argue at length to the effect that the FIR ought not, based on current evidence, to be denied associative status. Since the associative status of the FIR has 
not been in serious doubt for at least a decade (see, for example, reviews by Dengerink \& Taylor, 1971, and by Prokasy \& Kumpfer, 1973), the point is an obvious one. However, the organization of their discussion is such as to permit the erroneous impression that other investigators have, in recent years, employed various arguments to deny associative status to the FIR. Several of their comments merit classification.

The rationale cited by Furedy et al (1975) for discussing response definition is based on the suggestion made by Prokasy et al. (1973) that the SIR might be a more suitable measure of excitatory and inhibitory strengths than is the FIR. That suggestion was not, either directly or indirectly, designed to deny associative status to the FIR and therefore was not, as Furedy et al. state, one of "the primary issues which were alluded to in the Prokasy et al. (1973) article ..." Our comment on the SIR does recognize the fact that the FIR is influenced in ways (notaby by CS onset) which do not affect the SIR. This means that the effective range of FIR probability available to reflect associative influences is limited relative to that of the SIR.

A further misimpression is created from the Furedy et al. statement: "A final argument for the possible nonassociative status of the SCR FIR which needs consideration is Prokasy et al.'s (1973) claim, based on their earlier study (Prokasy \& Ebel, 1967) that FIR and SIR are not correlated." Contrary to the implication of the sentence, in neither cited study was the statistical independence of FIRs and SIRs employed to argue that FIRs are nonassociative. The statistical independence of FIR and SIR was employed solely as part of a conclusion that the two responses are not integral components of a single response waveform.

The major conclusion of the response definition section of the Furedy et al. paper is that researchers ought not to let analogies based on skeletal response systems "detemine $\mathrm{CR}$ specification in the electrodermal response system" (italics theirs). An unwary reader might gain the impression that somewhere it had been argued that such an analogy should determine CR specifcation in SCR conditioning. In spite of the number of investigators cited by Furedy et al., not a single one has ever made such a suggestion. Prokasy and Ebel (1967) did note a "striking parallel" between eyelid alpha response and FIRs but also provided evidence consistent with the view that the FIR is associative.

\section{REFERENCES}

Dengerink, H, A., Taylor, S. P. Multiple responses with diffexential properties in delayed galvanic skin response conditioning: A review. Psychophysiology, 1971, 8, 348-360.

Furedy, J. J. Explicitly-unpaired and truly-random CS-controls in human classical differential autonomic conditioning. Psychophysiology, 1974, 11, 308-314.

Furedy, J. J., Poulos, C. X., \& Schiffman, K. Contingency theory and classical autonomic excitatory and inhibitory conditioning: Some problems of assessment and interpretation. Psychophysiology, 1975, 12, 98-105.

Furedy, J. J., \& Schiffman, K. Test of the propriety of the traditional discriminative control procedure in Pavlovian electrodermal and plethysmographic conditioning. Journal of Experimental Psychology, 1971, 91, 161-164.

Prokasy, W. F. Classical eyelid conditioning: Experimental operations, task demands, and response shaping. In $W$. F. Prokasy (Ed.), Classical conditioning: A symposium. New York: Appleton-Century-Crofts, 1965.

Prokasy, W. F., \& Ebel, H. C. Three components of the classically conditioned CSR in human subjects. Journal of Experimental Psychology, 1967, 73, 247-256.

Prokasy, W. F., \& Kumpfer, K. L. Classical conditioning. In W. F. Prokasy and D. C. Raskin (Eds.), Electrodermanl activity in psychological research. New York: Academic Press, 1973.

Rescorla, R. A. Pavlovian conditioning and its proper control Procedures. Psychological Review, 1967, 74, 71-80.

Schiffman, K., \& Furedy, J. J. Failures of contingency and cognitive factors to affect long-interval differential Pavlovian autonomic conditioning. Journal of Experimental Psychology, 1972, 96, 215-218

\section{NOTES}

1. The values from the Furedy (1974) studies were supplied directly by Dr. Furedy.

2. Table 3 includes expectations because the exact figures were unavailable. Departures from expectation probably exist through random sampling and can be in either direction. Severe departures from these expectations would, of course, reflect nonrandom assignment.

(Received for publication February 3,1975 ; revision received May 8,1975 .) 